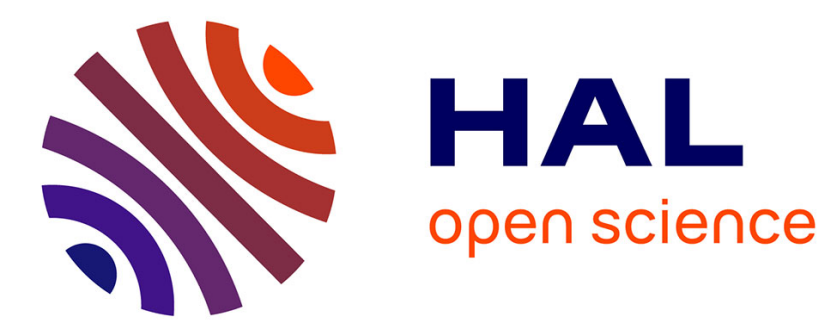

\title{
Émotions, droit et politique. Bilan et perspectives interdisciplinaires
}

Laurence Dumoulin, Cécile Vigour

\section{To cite this version:}

Laurence Dumoulin, Cécile Vigour. Émotions, droit et politique. Bilan et perspectives interdisciplinaires. Droit et Société, 2020, 105, pp.453-472. 10.3917/drs1.105.0453 . halshs-02975836

\section{HAL Id: halshs-02975836 \\ https://shs.hal.science/halshs-02975836}

Submitted on 8 Dec 2020

HAL is a multi-disciplinary open access archive for the deposit and dissemination of scientific research documents, whether they are published or not. The documents may come from teaching and research institutions in France or abroad, or from public or private research centers.
L'archive ouverte pluridisciplinaire HAL, est destinée au dépôt et à la diffusion de documents scientifiques de niveau recherche, publiés ou non, émanant des établissements d'enseignement et de recherche français ou étrangers, des laboratoires publics ou privés. 


\title{
ÉMOTIONS, DROIT ET POLITIQUE. BILAN ET PERSPECTIVES INTERDISCIPLINAIRES
}

\author{
Laurence Dumoulin, Cécile Vigour
}

Lextenso | « Droit et société »

2020/2 N 105 | pages 453 à 472

ISSN 0769-3362

Article disponible en ligne à l'adresse :

https://www.cairn.info/revue-droit-et-societe-2020-2-page-453.htm

Distribution électronique Cairn.info pour Lextenso.

(C) Lextenso. Tous droits réservés pour tous pays.

La reproduction ou représentation de cet article, notamment par photocopie, n'est autorisée que dans les limites des conditions générales d'utilisation du site ou, le cas échéant, des conditions générales de la licence souscrite par votre établissement. Toute autre reproduction ou représentation, en tout ou partie, sous quelque forme et de quelque manière que ce soit, est interdite sauf accord préalable et écrit de l'éditeur, en dehors des cas prévus par la législation en vigueur en France. Il est précisé que son stockage dans une base de données est également interdit. 
À propos 


\title{
Émotions, droit et politique. Bilan et perspectives interdisciplinaires
}

\author{
Laurence Dumoulin *, Cécile Vigour **
}

* Politiques publiques, Action politique, Territoires (PACTE), Sciences Po Grenoble-CNRS, BP 48, F-38040 Grenoble cedex 9. $<$ laurence.dumoulin@umrpacte.fr>

${ }^{* *}$ Sciences Po Bordeaux, 11 allée Ausone, F-33607 Pessac cedex.

$<$ c.vigour@sciencespobordeaux.fr>

\section{À propos de...}

- Bergman Bux Stina et Wettergren Åsa, Professional Emotions in Court: A Sociological Perspective, Londres : Routledge, 2018, 194 p.

- Bergman Blix Stina, Mack Kathy, Maroney Terry et Roach Anleu Sharyn (eds.), «Introducing an Interdisciplinary Frontier to Judging, Emotion and Emotion Work», Oñati Socio-Legal Series, 9 (5), 2019.

- Blondiaux Loïc et TraïNi Christophe (dir.), La démocratie des émotions. Dispositifs participatifs et gouvernabilité des affects, Paris: Presses de Sciences Po, 2018, $245 \mathrm{p}$.

- Milner Davis Jessica et RoACH Anleu Sharyn (eds.), Judges, Judging and Humour, Cham : Palgrave Macmillan, 2018, 335 p.

\section{Introduction}

Au croisement du droit, de la psychologie et de la sociologie, le courant de recherche Law and Emotion étudie la manière dont les émotions influencent le cadre et le raisonnement juridiques, la doctrine, l'activité et surtout les prises de décisions judiciaires ${ }^{1}$. Sont considérées les émotions suscitées par le droit, notamment le droit pénal (peur, vengeance...), les émotions ressenties par celles et ceux qui exercent la justice (l'empathie, la colère...), et celles qu'expriment ou pas les prévenus (remords, excuses, honte...), les victimes (souffrance, peur...) et parfois les témoins (angoisse, amertume...).

Pour une grande part d'entre eux, ces travaux s'inspirent de la psychologie et mettent l'accent sur les biais émotionnels inconscients qui pèsent sur la fabrique des décisions de justice. Ils prolongent ainsi une double tradition, très présente dans les travaux juridiques américains : celle des réalistes ${ }^{2}$ et celle de l'approche

1. Susan A. BANDES (ed.), The Passions of Law, New York : New York University Press, 2000.

2. Comme Jerome Frank qui a poussé loin l'idée de la subjectivité du juge, en proposant cette équation du jugement : $\mathrm{D}$ (la décision) = S (stimuli reçus par le juge) x P (sa personnalité), in Jerome Frank, Courts on 
psychologique du jugement ${ }^{3}$. Ces deux corpus ont contesté le postulat d'une délibération et d'un raisonnement juridiques purement rationnels et ouvert la voie à l'analyse de la dimension subjective et émotionnelle des décisions judiciaires. Depuis les années 1990, le courant Law \& Emotion se développe davantage en anglais qu'en français ${ }^{4}$, probablement en partie en raison de cet héritage et de l'intérêt traditionnellement plus important porté à la décision judiciaire en contexte de common law. S'il existe plusieurs manières de déployer une approche émotionnelle du droit, par exemple centrée sur les acteurs législatifs (lorsqu'ils adoptent des réformes pénales) ou judiciaires 5 , les décisions juridictionnelles sont un objet central de ce type d'approche, qui montre comment émotions et cognition interagissent précisément.

À défaut de dresser un état de l'art complet des approches émotionnelles du droit, cet $\grave{A}$ propos croise trois ouvrages et un dossier de revue parus en 2018-20196. Pour la plupart collectives, ces publications abordent une diversité de thèmes et de contextes empiriques; elles ont en commun de conférer aux émotions le statut de prisme d'analyse de ces différentes situations sociales et politiques. Rassemblant une diversité de regards disciplinaires, les trois publications anglophones sont marquées par une identité sociologique forte, que ce soit sur le plan des emprunts théoriques ou du rapport à l'empirie et aux méthodes employées, alors que tout un pan du courant Law \& Emotion est indexé sur des approches psychologiques, notamment expérimentales. Elles nous ont toutefois semblé particulièrement intéressantes parce qu'elles sont susceptibles d'être mises en lien avec des analyses francophones contemporaines, issues de la science politique et de la sociologie, avec lesquelles elles ne dialoguent pas - alors que certaines convergences sont manifestes - notamment parce qu'elles témoignent d'une même ambition de prendre au sérieux la dimension sociale des émotions.

Ces publications sont l'occasion de faire un rapide état des lieux de ce que nous savons aujourd'hui quant au rôle des émotions dans l'analyse des situations et interactions de justice. Parmi ces quatre publications, trois portent exclusivement sur la justice. Dans Professional Emotions in Court: A Sociological Perspective, Stina Bergman Blix et Åsa Wettergren, deux chercheuses suédoises, restituent une enquête approfondie sur les émotions au tribunal et le travail de gestion de celles-ci depuis les

Trial: Myth and Reality in American Justice, Princeton : Princeton University Press, 1949. En réalité, il faudrait également évoquer le réalisme scandinave car les deux entretiennent un «scepticisme [commun] quant à l'analyse de l'opération de décision judiciaire comme calcul logique à partir de règles ", voir Éric MILLARD, «Réalisme scandinave, réalisme américain. Un essai de caractérisation », Revus. Journal for Constitutional Theory and Philosophy of Law, 24, 2014, p. 85-86, <https://doi.org/10.4000/revus.2915>.

3. Depuis Georges Everson jusqu'aux behavioristes des années 1960, voir Thierry DeLPEUCH, Laurence Dumoulin et Claire DE GALEMBERT, Sociologie du droit et de la justice, Paris : Colin, 2014, chap. 2.

4. Lorsqu'elles le sont, c'est surtout dans le contexte de la cour d'assises que les émotions sont étudiées. Voir le dossier « Les émotions dans le prétoire », Les Cahiers de la justice, 1, 2014.

5. Pour une synthèse en français, voir Alexandre FlÜCKIGER, Robert RotH et Christian-Nils RoBERT (dir.), Droit et émotions : le rôle des émotions dans les processus de régulation juridique et sociale, Genève : CETEL, 2010, p. 6-9.

6. Un dossier consacré à « l'ethnographie politique et comparative des émotions », co-dirigé par Stéphanie Dechézelles et Christophe Traïni, a aussi été publié dans la Revue internationale de politique comparée en 2018, vol. 3-4. Il met l'accent sur l'apport de l'observation ethnographique dans le saisissement du rôle des émotions dans des types variés d'interactions, y compris au cours de la chaîne pénale. 
coulisses, le travail sur les dossiers, jusqu'à l'audience, en passant par la prise de décisions. Nous retrouvons ces autrices parmi les directrices du dossier «Introducing an Interdisciplinary Frontier To Judging, Emotion and Emotion Work», publié par la revue de l'Institut de sociologie du droit d'Oñati. Ce dossier comprend quinze articles, organisés en quatre thèmes: "Perspectives théoriques, culturelles et historiques", «Tensions autour de l'idéal judiciaire de détachement [dispassion]", "Les dynamiques sociales des émotions dans le jugement ", et "Les méthodes de recherche et pratiques judiciaires". Le troisième opus resserre la discussion sur les juges et l'humour. Cet ouvrage dirigé par deux universitaires australiennes, Jessica Milner Davis et Sharyn Roach Anleu, elles aussi contributrices dans le précédent dossier, comprend neuf chapitres organisés en trois parties : "L'humour chez les juges"; "L'utilisation de l'humour par les juges»; "Les décisions judiciaires relatives à l'humour ». L'approche par les émotions est présente surtout dans la seconde partie. Le dernier ouvrage La démocratie des émotions, dirigé par Loïc Blondiaux et Christophe Traïni et inscrit en science politique, porte sur la dimension émotionnelle de la participation politique. Les huit chapitres qui le constituent sont organisés en trois parties qui traitent successivement de la dimension inclusive des émotions en politique, du façonnage institutionnel des sensibilités citoyennes ainsi que de la maîtrise des émotions dans l'ingénierie participative. Il est question de justice principalement dans le chapitre sur les expériences émotionnelles en cour d'assises (Célia Gissinger-Bosse, 2018) ${ }^{7}$. La présente note abordera surtout ce chapitre et l'introduction générale.

Ces quatre publications remettent en cause l'idée reçue du caractère inapproprié, voire perturbateur des émotions pour l'analyse scientifique des phénomènes sociaux et politiques. Traditionnellement, les juristes ont eu tendance à « construire les émotions comme "autres" que le droit " 8 , à la fois parce qu'elles étaient extérieures aux règles juridiques et à l'activité de production des règles et parce qu'ils ont fréquemment associé la régulation juridique à la raison et à la rationalité. Or, les études Law and Emotion se fondent sur la remise en cause de ce double a priori. Leur agenda de recherche a d'abord porté sur l'analyse des émotions qui pervertissent la mise en œuvre du droit - dans une approche elle-même normative - puis à travers un renversement de perspective, sur l'analyse de la manière dont les émotions peuvent enrichir la compréhension du droit et de son exercice par des acteurs, professionnels ou non ${ }^{9}$. Partant du principe selon lequel les émotions se manifestent chez les juges comme chez n'importe quel être humain et qu'elles affectent toutes les fonctions corporelles, y compris cognitives, il est établi que «l'émotion judiciaire, inscrite dans des contextes culturels et sociaux, scénarise [script] quelles expériences émotionnelles sont acceptables et peuvent être exprimées [display] » 10.

7. Les références indiquées entre parenthèses renvoient aux articles et contributions des ouvrages discutés. Ceux-ci sont répertoriés en bibliographie, à la fin de cet $\grave{A}$ propos.

8. Bettina LANGE, "The Emotional Dimension in Legal Regulation », Journal of Law and Society, 29, 2002, p. 199. Toutes les traductions sont le fait des autrices.

9. Susan A. BAndes et Jeremy A. Blumenthal, "Emotion and the Law ", Annual Review of Law and Social Science, 8, 2012, p. 161-181.

10. Sharyn RoACH ANLeu et Kathy MACK, Performing Judicial Authority in the Lower Courts, Londres : Palgrave, 2017. 
Les contextes culturels et le milieu professionnel influencent les attentes relatives à ce qu'est un bon magistrat, y compris sur le plan émotionnel. Une vague de travaux empiriques s'est focalisée sur " les événements, les circonstances ou les processus » judiciaires dans lesquels des émotions surgissent ou sont mobilisées. Mais, quelles que soient les disciplines, les émotions judiciaires apparaissent aujourd'hui encore comme une boîte noire qu'il s'agit d'ouvrir davantage (Terry A. Maroney, 2019, p. 802). Les ouvrages et dossier ici discutés envisagent les émotions comme des ressources dans l'activité judiciaire plutôt que comme un obstacle à une bonne justice. L'enjeu est de dépasser la dichotomie entre rationalité et détachement, d'une part, et émotions, d'autre part, pour montrer comment celles-ci se manifestent, comment elles contribuent à la fabrique de l'intime conviction (C. GissingerBosse, 2018, p. 125) et quelles stratégies de régulation des émotions sont mises en œuvre par les professionnels (S. Bergman Blix et Å. Wettergren, 2018). En effet, si le droit et la justice continuent en partie de reposer sur le «script d'une justice détachée » 11 et la fiction d'un juge sans passion (S. Bergman Blix et al., 2019, p. 552) dont les émotions sont systématiquement mises sous silence par le rituel judiciaire (C. Gissinger-Bosse, 2018, p. 122), force est de constater que certaines émotions se manifestent même là où cela est $a$ priori pensé comme exclu, comme par exemple différentes formes d'humour dans les salles d'audience (J. Milner Davis et S. Roach Anleu, 2018, p. 15) ou dans les «rituels d'interactions " 12 en délibération de jury (Meredith Rossner, 2019). Les émotions sont de ce fait constituées en objet de recherche pluridisciplinaire, dont l'analyse implique chercheurs et praticiens.

Or, cette perspective manque encore de concepts pertinents et langages distincts pour démêler les expériences émotionnelles et en rendre compte. Certains auteurs engagent donc un travail de type analytique, théorique et empirique que nous allons restituer successivement pour aborder ensuite deux problématiques qui parcourent les quatre ouvrages et revue chroniqués : les émotions et leur régulation en contexte de travail d'une part; la dimension politique des émotions d'autre part.

\section{I. Étudier les émotions : quels concepts et distinctions analytiques?}

Dans un précédent article, C. Traïni soulignait l'importance de distinguer sentiment et émotion : «Il paraît crucial de réserver le terme "émotion" afin de désigner l'activité des individus dès lors qu'elle relève de ce travail de sélection et de transformation des informations par la conscience réfléchie. Contrairement à ce que pourrait nous laisser penser le langage courant, lorsqu'il l'érige en l'antithèse de la raison, l'émotion ne peut se manifester en l'absence d'un degré minimal aussi bien de réflexivité que de compréhension intersubjective. [...]. S'émouvoir implique une évaluation cognitive préalable, d'une part de la situation, d'autre part du vécu subjectif qu'elle provoque, et ce, afin de rendre intelligibles les réactions affectives qui étreignent le corps ${ }^{13}$.» Ces réactions

11. Terry A. Maroney, "The Persistent Cultural Script of Judicial Dispassion", California Law Review, 99 (2), 2011, p. 629-681, en ligne : <https://doi.org/10.15779/Z38K98M>.

12. Randall Collins, Interaction Ritual Chains, Princeton : Princeton University Press, 2004.

13. Christophe TraïnI, «Des sentiments aux émotions (et vice-versa). Comment devient-on militant de la cause animale ? ", Revue française de science politique, 60 (2), 2010, p. 339-340. 
affectives brutes, non encore nommées et sans exercice de la réflexivité sont ce que C. Traïni désigne par le terme de sentiment.

Dans le sillage de Jack Barbalet 14, Stina Bergman Blix et Åsa Wettergren (2018), quant à elles, distinguent les background et foreground emotions, soit le fait d'être traversé ou non par les émotions concernées. Les "foreground emotions» sont de fortes émotions qui peuvent intervenir dans l'activité professionnelle ou l'interrompre. Elles requièrent l'attention et l'implication du sujet, tandis que les background emotions, caractérisées par une faible expressivité, ne sont pas tournées vers l'intérieur du sujet: ces émotions sont mobilisées par la cognition dans une perspective rationnelle et instrumentale. Ces deux concepts permettent de comprendre la mise sous silence des émotions pourtant si présentes, même implicitement, dans les récits que les acteurs judiciaires donnent de leur quotidien, ainsi que le caractère routinier des modes de gestion des émotions mis en œuvre dans l'institution judiciaire. Le livre de S. Bergman Blix et Å. Wettergren (2018, p. 13) se focalise sur «les background emotions qui prennent place dans les interactions ordinaires au cœur du travail juridique ». L'enjeu est d'en apprendre davantage sur la mobilisation de ces compétences professionnelles tacites, en s'interrogeant sur les manières dont les émotions sont actives dans la justice pénale. Ces autrices s'intéressent particulièrement à leur incorporation au cours des processus de socialisation et à travers l'expérience des professionnels : la notion anglophone d'habituation rend compte de cette intériorisation (p. 19). Avec l'expérience, les magistrats ressentent moins fortement les émotions, ils connaissent les règles quant à leur expression. Selon les cas, la routinisation conduit à une distanciation émotionnelle (par exemple lors de la lecture de témoignages), tandis que les acteurs judiciaires ont besoin de maintenir une présence émotionnelle sans être submergés par les affects lors des interactions comme à l'audience.

T. Maroney (2019, p. 801) se réfère au triptyque de l'expérience émotionnelle, de la régulation des émotions et des effets émotionnels. La régulation judiciaire des affects correspond aux «manières dont les magistrats s'efforcent de gérer leurs propres expériences émotionnelles, comme celles des autres (avocats ou justiciables), en fonction de leurs contraintes professionnelles". Les effets émotionnels sont les conséquences de ces expériences et processus de régulation sur l'exercice de la justice et le bien-être des magistrats. C'est pourquoi les émotions relèvent « de l'intra et de l'interpersonnel », de l'individuel et du collectif (cf. aussi S. Bergman Blix et Å. Wettergren, 2018).

Les recherches sur les émotions se focalisent de plus en plus sur l'émotion appréhendée comme un phénomène non seulement individuel, mais comme une composante essentielle des dynamiques sociales et institutionnelles 15. C'est un point de convergence entre l'approche de science politique développée par L. Blondiaux et C. Traïni et les publications anglophones ici chroniquées. "Dire que les émotions sont sociales, c'est affirmer qu'elles ne sont pas essentiellement la résultante d'états intérieurs, d'états de conscience - qui, à leur tour, motiveraient des actions, mais l'effet émergent d'une activité dont le déroulement est inséparable des traits de la

14. Jack BARBALET, «Emotions Beyond Regulation: Backgrounded Emotions in Science and Trust », Emotion Review, 3 (1), 2011, p. 39.

15. Susan A. BANDES et Jeremy A. Blumenthal, « Emotion and the Law », article cité. 
situation dans laquelle elles se déploient ${ }^{16}$. » Les émotions relèvent donc de l'interprétation d'une situation et prennent sens dans une relation : "Nommer l'émotion consiste, comme on l'a dit, à interpréter une situation ; ce n'est pas fournir des motifs à nos actions (“j'ai peur, donc je m'enfuis”, “je suis enthousiaste, donc j'applaudis”), mais c'est "relier un comportement à un arrière-plan complexe dans lequel il est accompli, et, ce faisant, rendre les actions humaines intelligibles" 17. " Mais, dire que les émotions sont sociales, c'est affirmer aussi que «le sens de l'émotion ne dépend pas de celui qui l'exprime, ni d'ailleurs de celui qui la reçoit, mais d'un processus de coordination entre eux " ${ }^{18}$. L'émotion est ainsi le produit des ajustements entre différents acteurs, dans des contextes sociaux, institutionnels ou politiques donnés. La composante interactionnelle des émotions et les effets de coordination de l'action collective sont donc au cœur de l'analyse des textes discutés.

\section{Quels cadres théoriques pour appréhender les émotions ?}

Une grande diversité de cadres disciplinaires et théoriques est mobilisée pour prendre en compte les émotions. S'agissant de l'humour par exemple, J. Milner Davis et S. Roach Anleu recensent une théorie générale essentialiste, une théorie de l'humour comme forme de la domination sociale, diverses approches de psychologie, d'anthropologie et de sociologie linguistiques (2018, p. 5 et suiv.), et encore, elles n'incluent que des analyses interprétatives. Les sciences cognitives sont peu présentes. Seul un article du dossier publié par la revue d'Oñati (Maria Gendron et Lisa F. Barrett, 2019) se focalise sur les apports des neurosciences et de la psychologie comportementale, tandis que T. Maroney (2019) se réfère à d'autres expériences en psychologie.

En outre, plusieurs publications recourent à la notion de «formalisation des émotions », mais seuls L. Blondiaux et C. Traïni la définissent et l'appuient explicitement sur la sociologie historique de Norbert Elias ${ }^{19}$. Ils entendent par formalisation « la soumission de l'expression des émotions au respect des règles qui dessinent un accord - certes plus ou moins bien partagé parmi les participants - sur ce qui apparaît convenable dans les circonstances observées» (L. Blondiaux et C. Traïni, 2018, p. 20).

Toutefois, certains cadres théoriques marquent plus fortement les ouvrages recensés, comme la sociologie interactionniste, et les notions goffmaniennes de cadre et de performance, via des comparaisons récurrentes avec le théâtre (S. Bergman Blix et Å. Wettergren, 2018a, b ; C. Gissinger-Bosse, 2018).

Pour S. Bergman Blix et Å. Wettergren (2018a, p. 20), la justice est définie d'emblée comme "une réalisation interactionnelle commune de différents professionnels du droit agissant dans le tribunal ». Celui-ci est appréhendé comme un cadre interaction-

16. Jean-Philippe Heurtin, «L'enthousiasme du Téléthon », in Christophe TrAïNI (dir.), Émotions... Mobilisations !, Paris : Sciences Po, 2009, p. 104.

17. Erro BEDFord, «Emotions and Statements about Them », in Rom HARRÉ (ed.), The Social Construction of Emotions, Oxford : Blackwell, 1986, p. 15-31, cité par Jean-Philippe Heurtin, ibid., p. 105.

18. Jean-Philippe Heurtin, « L'enthousiasme du Téléthon », op. cit., p. 105.

19. Norbert EliAs et Éric DUNNING, Sport et civilisation. La violence maîtrisée, Paris : Fayard, 1994. 
nel qui structure les expériences ${ }^{20}$, marqué par le passage entre des moments publics accomplis sur la scène judiciaire et d'autres effectués en coulisses ${ }^{21}$. À ce cadre interactionnel sont attachées des évaluations morales quant à la manière dont les personnes doivent se comporter (p. 22), les émotions qu'elles peuvent et/ou doivent manifester ou non. Le « cadrage judiciaire émotionnel et cognitif » [emotional-cognitive judicial frame] agit comme « un script qui contient et restreint le comportement " [ restricted behavioral script»] des professionnels du droit. Les émotions et leur gestion sont centrales dans l'établissement d'une routine professionnelle conforme à ce cadre. En témoigne le fait que les professionnels éprouvent de la fierté lorsqu'ils parviennent à les maîtriser et considèrent $a$ contrario l'embarras comme un signal d'alerte.

Le cadrage judiciaire des émotions s'exprime différemment chez les juges et procureurs, ce dont rend compte la notion de profils émotionnels. Selon S. Bergman Blix et Å. Wettergren (2018, p. 23), bien qu'insérés dans un même cadre judiciaire émotionnel et cognitif, les juges et procureurs suédois relèveraient "de profils émotionnels professionnels » distincts, car leur mode de sélection et leur rôle dans le système judiciaire et leur travail diffèrent. Les uns et les autres disposeraient de techniques en partie différentes pour gérer les expériences émotionnelles fortes, cadrées par le rituel judiciaire (cf. ci-dessous).

Les notions d'interactions sociales et de rituels sont aussi centrales dans les chapitres 1, 5 et 6 de l'ouvrage collectif sur l'humour (J. Milner Davis et S. Roach Anleu, 2018 ; S. Bergman Blix et Å Wettergren, 2018b ; S. Roach Anleu et K. Mack, 2018). Dans ces chapitres, les audiences sont analysées comme des interactions rituellement réglées, dont l'accomplissement repose sur la manière dont les individus interagissent en s'appuyant sur des rôles institutionnels. Trois articles fondent leur cadre théorique sur le prisme interactionnel et, à partir d'enquêtes par observations, mettent en évidence ce que les interactions "font " aux procédures judiciaires (S. Bergman Blix et Å. Wettergren, 2019 ; M. Rossner, 2019 ; Leslie. J. Moran, 2019). L'approche interactionnelle traverse également plusieurs articles de la revue Oñati Socio-Legal Series qui étudient la manière dont le juge influence les émotions ressenties par les autres acteurs et leur performance (Bridgette Toy-Cronin, 2019 ; Tracey Booth, 2019).

Enfin, les recherches relatives à la justice procédurale 22 , qui reposent sur une approche de psychologie juridique, sont mentionnées par plusieurs travaux recensés ici, notamment Steve Leben (2019) et ceux sur l'humour. Étudier le lien entre (in)justice procédurale, émotions et attitudes ultérieures d'obéissance ou non à la loi est toutefois difficile puisque mettre en évidence ces liens de causalité requiert un protocole d'enquête longitudinal 23 .

20. Erving GoffMAn, Frame Analysis. An Essay on the Organization of Experience, Boston, MA : Northeastern University Press, 1974, p. 13.

21. ID., The Presentation of Self in Everyday Life, New York : Doubleday, 1959.

22. Tom TyLER, Why People Obey the Law, Londres : Yale University Press, 1990. Kristina MurPHY et Tom TYLER, «Procedural Justice and Compliance Behaviour: The Mediating Role of Emotions ", European Journal of Social Psychology, 38, 2008, p. 652-668.

23. Julie Barkworth et Kristina MurPhy, «Procedural Justice Policing and Citizen Compliance Behaviour: The Importance of Emotion », Psychology, Crime \& Law, 21 (3), 2015, p. 254-273. 


\section{Pour une approche empirique au plus près des pratiques}

De manière générale, étudier les émotions vécues et leurs effets présente des défis méthodologiques et d'administration de la preuve 24 ne serait-ce que pour les identifier, les analyser et les coder, a fortiori concernant les background emotions (S. Bergman Blix et al., 2019, p. 556). Il convient de ne pas confondre les états affectifs « exprimés » et «éprouvés» (L. Blondiaux et C. Traïni, 2018, p. 17). Dans le cas de l'humour, il est important de distinguer entre le fait de lancer un trait d'humour, la perception cognitive du public (l'avoir saisi) et l'expérience affective, la réaction comportementale qu'il suscite (le rire, la vexation, la colère, etc.). Comment objectiver le trait d'humour et par qui : celui qui s'y livre, le chercheur qui observe et / ou les autres participants? Le contexte culturel, social et individuel de réception est essentiel car l'humour n'est pas drôle en soi (J. Milner Davis et S. Roach Anleu, 2018, p. 17). Quels indicateurs retenir dans l'observation pour effectuer un relevé des émotions? Quelles questions poser en entretien pour accéder à l'expression d'émotions par des professionnels du droit imprégnés de normes visant à leur intériorisation, voire à leur inhibition?

Différentes méthodologies sont utilisées et souvent combinées entre elles, qu'il s'agisse d'un éventail de méthodes qualitatives ou d'un croisement quali-quanti. T. Maroney (2019) en livre un panorama descriptif, mais utile que nous restituons ici.

En ce qui concerne les méthodes qualitatives, S. Bergman Blix et Å. Wettergren (2018a) ont associé la réalisation de presque 150 entretiens et débriefings d'audience avec des juges, parquetiers et avocats ; l'observation de 300 audiences dans lesquelles ont été analysés le langage corporel, les expressions faciales, les regards et le vocabulaire utilisé ; le suivi en continu des magistrats dans leur travail quotidien, au bureau, en salle d'audience, lors des repas et pauses (méthode dite du shadowing ${ }^{25}$ ) ; la " participation émotionnelle » (p. 21), c'est-à-dire la réflexivité des chercheuses par rapport à leurs propres émotions considérées comme un levier d'analyse concernant les situations ou les personnes qu'elles observent. Ces autrices abordent en entretien les aspects les plus agréables ou déplaisants de l'activité des enquêtés ; les motifs de fierté dans leur travail ainsi que ce qui, à leurs yeux, fait d'eux.elles un·e bon·ne juge ou un·e bon·ne procureur.e. T. Maroney dans ses recherches 26 a quant à elle recours à des questions du type : "Pouvez-vous me raconter un moment où vous avez ressenti de la colère au travail ? ", "Quand vous ressentez de la colère, y a-t-il quelque chose que vous essayez de faire pour affronter ce sentiment?», "Parlez-vous avec d'autres juges des défis que représentent les émotions ressenties au travail ?» Enfin, C. Gissinger-Bosse a observé des procès et réalisé des entretiens semi-directifs avec des anciens jurés et des présidents de cours

24. Helena Flam et Jochen KLERES (eds.), Methods of Exploring Emotions, Londres : Routledge, 2015.

25. Seonaidh McDonALD, «Studying Action in Context. A Qualitative Shadowing Method for Organizational Research », Qualitative Research, 5 (4), 2005, p. 455-473.

26. Terry A. MARONEY, « Emotional Regulation and Judicial Behavior», California Law Review, 99 (6), 2011, p. 1481-1551, en ligne : <https://ssrn.com/abstract=1785616> ; ID., « Angry Judges », Vanderbilt Law Review, 65 (5), 2012, p. 1205-1286. 
d'assises. Toutes les recherches de l'ouvrage dans lequel son chapitre est publié sont de type ethnographique (L. Blondiaux et C. Traïni, 2018).

Le croisement de méthodes qualitatives et quantitatives a été pratiqué dans la recherche de S. Roach Anleu et K. Mack (2017) centrée sur les émotions ressenties par les juges australiens et sur leur gestion lors des interactions au tribunal 27 . Ont été associés un questionnaire, des observations d'audience (par deux observateurs pour s'assurer de la fiabilité du codage des émotions) et 38 entretiens semi-directifs. Ce protocole a mis en évidence un contraste entre ce que les magistrats interrogés affirment et ce que les chercheurs observent. Le chapitre 6 du livre collectif sur les juges et l'humour livre quelques résultats tirés des seules données qualitatives de cette recherche.

Des échelles de psychologie sont aussi utilisées dans une perspective de recherche-action (T. Maroney, 2019). Ainsi un projet australien sur le niveau de stress judiciaire recourt à l'échelle DASS-21 (Depression, Anxiety and Stress Scales 21 ; Schrever et al., 2019, p. 142). Il combine court et long questionnaires ; entretiens semi-directifs.

Enfin, diverses méthodes se focalisent sur l'évaluation du rôle des émotions dans la fabrique des décisions de justice. Toutefois, cette dimension, cruciale dès l'origine du courant Law and Emotion, est peu présente dans les publications recensées ici, excepté dans la contribution de M. Gendron et L. Barrett (2019). À partir d'analyses statistiques, deux politistes soutiennent qu'avoir élevé une fille influencerait les décisions des juges américains des cours d'appel fédérales concernant les affaires relatives aux droits des femmes ${ }^{28}$. Élever une fille aiderait à cultiver l'empathie dans ses dimensions cognitives et émotionnelles, et cette empathie ferait une différence dans la façon dont les juges, a fortiori les hommes conservateurs, statuent sur les affaires. D'autres recherches portant sur l'influence des émotions sur les jugements procèdent par expérimentation ${ }^{29}$. Les juges lisent des scénarios fictifs et tranchent des questions de droit très techniques. Il en ressort que les juges étudiés sont enclins à interpréter la loi d'une manière plus favorable aux parties sympathiques et plus défavorable aux parties antipathiques. Les expériences fournissent la preuve que les décisions juridiques des juges pourraient être façonnées en partie par l'«heuristique de l'affect», c'est-à-dire un ressenti d'appréciation positive ou négative qui motive les choix ensuite rationalisés, relatifs à la manière d'interpréter des sources de droit ambiguës. Un lien causal apparaît clairement en ne faisant varier qu'un paramètre. En revanche, ces expérimentations interrogent sur les possibilités de généralisation des résultats, puisque, dans la pratique, de multiples facteurs se combinent.

27. Un article est disponible en français : Sharyn ROACH ANLEU et Kathy MACK, « Le quotidien des magistrats et le travail émotionnel ", Les Cahiers de la justice, 1, 2014, p. 27-48.

28. Adam N. GlynN et Maya SEN, «Identifying Judicial Empathy: Does Having Daughters Cause Judges to Rule for Women's Issues? », American Journal of Political Science, 59 (1), 2014, p. 37-54.

29. Andrew J. Wistrich, Jeffrey J. RACHLInSKI et Chris Guthrie, « Heart Versus Head: Do Judges Follow the Law or Follow Their Feelings?», Texas Law Review, 93 (4), 2015, p. 855-923, en ligne : <http://texaslawreview.org/ wpcontent/uploads/2015/08/Rachlinski-93-4.pdf> - cités par Terry A. MARONEY, « Empirically Investigating Judicial Emotion », Oñati Socio-Legal Series [en ligne], 9 (5), 2019, p. 799-830. 


\section{Ce que nous apprennent les expériences émotionnelles}

Deux lignes de force traversent les quatre ouvrages et revue présentés ici : les émotions au travail et le travail sur les émotions d'une part ; la dimension politique des émotions d'autre part.

\section{IV.1. Les émotions au travail et le travail sur les émotions}

Le cadre judiciaire remplit une fonction disciplinaire et performative ${ }^{30}$ en excluant les émotions de la sphère professionnelle (S. Bergman Blix et Å. Wettergren, 2018a, p. 163). Pourtant, comme le montrent les ouvrages et articles étudiés ici, les processus émotionnels font partie du travail judiciaire ; c'est pourquoi les professionnels développent des techniques de gestion des émotions.

\section{La gestion des émotions, socle de l'objectivité et de l'impartialité}

De nombreux travaux fondés sur des méthodologies ethnographiques, notamment dans les pays de common law, ont établi qu'en dépit de l'idéal de distance émotionnelle, les juges manifestaient de multiples émotions à l'audience : condescendance, dureté, ennui... ${ }^{31}$ S. Bergman Blix et Å. Wettergren (2018a) défendent la thèse selon laquelle l'objectivité et l'impartialité reposent en pratique non pas sur la négation des émotions, mais sur leur gestion.

Dans le chapitre 4, consacré à l'audience, elles analysent comment la dramaturgie de l'État de droit est conçue pour canaliser les émotions. «Rendre une justice objective de manière concertée implique des professionnels qu'ils communiquent les émotions entre eux, à la fois tacitement et ouvertement» (p. 30). Pour minimiser leur expression, les magistrats recourent à un accordage émotionnel (emotional toning), " ajustement qui conduit à une incongruence intériorisée entre l'expérience et l'émotion exprimée» (p. 111). Quand la discordance est trop forte entre la description juridique d'un crime et sa réalité émotionnelle, les professionnels du droit ressentiraient des foreground emotions, parfois en décalage avec les attentes des profanes.

Au chapitre 6, S. Bergman Blix et Å. Wettergren montrent à quel point l'objectivité et l'impartialité sont profondément émotionnelles, car les normes morales du cadre judiciaire cognitif et émotionnel orientent l'action des professionnels quant à la fierté (de «bien faire») ou à la honte (de «mal faire»). Être objectif signifie "ne pas être influencé par des sentiments ou des opinions personnelles dans la considération ou la représentation de faits " (p. 141) ; être impartial, traiter quelqu'un équitablement. L'objectivité se réalise dans des processus situés d'interactions impliquant la gestion des émotions. En Suède, les juges se focalisent sur l'impartialité (en équilibrant les émotions exprimées à l'égard des victimes et auteurs), les procureurs sur l'objectivité qui implique un dialogue interne réflexif : ils s'efforcent d'équilibrer engagement et détachement, puisqu'ils doivent enquêter à charge et à décharge, tout en représentant la société à l'audience.

30. Michel Foucault, Surveiller et punir, Paris : Gallimard, 1975. William RedDY, The Navigation of Feeling. A Framework for the History of Emotions, Cambridge : Cambridge University Press, 2001.

31. Sharyn RoACH AnLeU et Kathy MACK, " Magistrates' Every Day Work and Emotional Labour », Journal of Law and Society, 32, 2005, p. 590-614. 
S. Roach Anleu et K. Mack (2019) se penchent, à partir d'une étude de cas, sur la manière dont une juge australienne gère ses émotions. Elles montrent aussi que les efforts pour garantir l'impartialité constituent un instrument de gestion des émotions qui façonne les frontières du jugement, des émotions et du travail sur les émotions. Ce dernier apparaît central dans l'exercice de la justice.

Enfin C. Gissinger-Bosse (2018, p. 128) établit que les émotions exprimées par les témoins, victimes et accusés ont une fonction particulière pour les jurés. En leur permettant de mieux évaluer la véracité du récit, et de se représenter plus concrètement ce que les personnes éprouvent ou ont éprouvé, elles contribuent activement à la formation de leur intime conviction.

Parfois, les émotions sont aussi l'occasion d'exprimer des jugements moraux. Selon S. Bergman Blix et Å. Wettergren (2018), les juges suédois manifestent certes des émotions, mais peu, voire pas de jugements moraux, à la différence des juges de common law aux États-Unis, selon les travaux de T. Maroney ${ }^{32}$. Dans sa comparaison entre la France et le Québec, une région nord-américaine principalement de droit écrit, Émilie Biland 33 montre aussi que les juges aux affaires familiales n'y manifestent pas de la même manière des jugements moraux. En Suède, extérioriser de tels jugements serait très peu acceptable, au motif que l'objectivité et l'impartialité de la justice seraient menacées.

\section{Des techniques de gestion des émotions individuelles plus que collectives}

S. Bergman Blix et Å. Wettergren (2018a, chapitre 3) identifient de multiples techniques de gestion individuelle et collective des émotions mobilisées par les magistrats. De manière informelle, certains d'entre eux s'épanchent auprès de leurs conjoints ou collègues en sortant d'une audience - un procédé (l'emotion ventilation) que les femmes mentionnent davantage que les hommes. Toutefois, les juges perçoivent ce besoin de parler comme un signe de vulnérabilité. Certains tentent aussi de limiter le recours aux photos et vidéos qui pourraient susciter trop d'émotions à l'audience; mais dans ce cas, les procureurs s'y opposent parfois au motif que les images influencent le verdict. Les magistrats sont pris en étau entre la nécessité de s'endurcir et le fait d'être capable d'empathie. Ce que les autrices désignent par le terme othering (que l'on pourrait tenter de traduire par le "syndrome de l'autre» ou la technique du «je ne parle pas en mon nom ») et qui consiste en un déplacement des peurs, attribuées à des caractéristiques personnelles, familiales ou sociales (les jeunes collègues, le fait d'être parent..., p. 67) ; par exemple, une juge organise un débriefing qu'elle décrit comme au bénéfice des assesseurs et du greffier. Le «ventriloquisme » 34 , lui, renvoie au fait de parler de manière factuelle des émotions sans le reconnaître, ni les nommer, par exemple en demandant à ses collègues : «Comment ferais-tu s'il se produisait telle situation?» (p. 83). Cette technique permet

32. Terry MARONEY, « Angry Judges », article cité.

33. Émilie Biland, Gouverner la vie privée. L'encadrement inégalitaire des séparations conjugales en France et au Québec, Lyon : ENS Éditions, 2019.

34. Charlotte Bloch, Passion and Paranoia. Emotions and the Culture of Emotion in Academia, Londres : Routledge, 2016. 
d'évoquer une affaire traumatisante, sans rompre avec les règles relatives au cadre interactionnel de l'échange judiciaire. Les magistrats, juges surtout, soutiennent davantage un·e de leurs collègues, si la demande porte sur les aspects juridiques et formels que sur le contenu émotionnel.

Une autre forme de "ventriloquisme » s'observe dans les cultures de la plaisanterie : l'humour noir met en évidence les tensions et les émotions sombres sans en parler directement. Comme le souligne l'ouvrage dirigé par J. Milner Davis et S. Roach Anleu, l'humour est en soi une façon de gérer ses émotions. Revenir lors des déjeuners et pauses, sur des séquences d'audience, diffère le moment de la réaction; ces discussions informelles assurent aussi une socialisation des plus jeunes (S. Bergman Blix et A. Wettergren, 2018b, p. 200). L'humour constitue aussi une ressource organisationnelle pour les juges australiens (S. Roach Anleu et K. Mack, 2018, p. 146) : il contribue à l'accomplissement de certains buts organisationnels, comme effectuer certaines tâches quotidiennes (fixer une date pour une audience future) ainsi qu'à réaffirmer les frontières interprofessionnelles entre les types de participants à l'audience (juge, procureur, avocat). Les réactions à l'humour volontaire ou non, les techniques employées lorsque l'envie de rire émerge dans des circonstances peu appropriées sont individuelles, mais stéréotypées : éviter les échanges de regards, se concentrer sur son ordinateur, etc. (S. Bergman Blix et Å. Wettergren, 2018b, p. 196).

Pour S. Bergman Blix et $\AA$. Wettergren (2018a, p. 73), travailler dur et de nombreuses heures, ne jamais se plaindre y compris des émotions ressenties vis-à-vis de la grande misère humaine ou d'affaires violentes..., faire face à ses émotions sont autant de composantes d'une culture d'autodiscipline qu'elles qualifient de «culture téflon». En Suède, c'est l'individu qui est identifié comme le lieu de gestion des émotions. L'incapacité à surmonter les difficultés émotionnelles, la pression au travail et le stress est interprétée comme le signe d'une inaptitude à exercer ce métier. Il en résulte de fortes résistances vis-à-vis des discussions psychologiques. Même si certain·e·s juges "prennent leurs distances avec l'idéal du juge sans émotions, [ils·elles] y souscrivent en tant que prérequis professionnel " (ibid., p. 75). Les procureurs suédois seraient plus réflexifs quant à la manière dont ils gèrent leurs émotions dans les cas difficiles ; ils en parleraient plus facilement entre eux de manière informelle et collégiale; leurs relations avec de nombreux autres professionnels, dont les travailleurs sociaux, expliqueraient leur plus grande ouverture aux débriefings. Ainsi juges et procureurs ont des profils émotionnels en partie distincts. Pour les acteurs judiciaires, la verbalisation des émotions (emotion talk) ne relève pas de la sphère professionnelle; elle est associée au moi privé (private self), (p. 163). Toutefois, selon les autrices, une telle dichotomie ne correspond ni aux expériences, ni aux pratiques judiciaires; la gestion collective des émotions relève selon elles de la responsabilité de l'institution judiciaire.

\section{IV.2. Des émotions politiques}

Les émotions peuvent être qualifiées de politiques à la fois parce que leur expression et leur gestion sont liées aux inégalités de pouvoirs et de statuts, et parce qu'elles conditionnent la légitimité de l'exercice de la justice aux yeux du public. 


\section{La maîtrise du travail émotionnel inégalement répartie selon les pouvoirs et statuts professionnels}

Les « écarts [dans la maîtrise du travail émotionnel] semblent difficilement dissociables des inégalités et des statuts socioprofessionnels qui structurent le monde social. Les plus habiles en matière de modulation des émotions appartiennent généralement aux catégories sociales les plus favorisées : magistrats » (L. Blondiaux et C. Traïni, 2018, p. 33). Ils exercent un «contrôle émotionnel » 35 sur eux-mêmes et sur les autres, qui exprime un pouvoir entendu dans un double sens : wébérien dans des relations asymétriques, une personne a des ressources pour commander aux autres - et foucaldien - les relations de pouvoir façonnent la subjectivité à travers les émotions. Plus encore dans une situation comme celle de l'audience où « la loi encadre [la] mise à distance des émotions, et le président d'audience est garant de ce contrôle»(C. Gissinger-Bosse, 2018, p. 122). Les citoyens apprennent «à s'émouvoir comme il faut» (ibid., p. 142-143) : certaines émotions sont autorisées, et même appréciées par les jurés et magistrats. En revanche, il est attendu de ces mêmes jurés - mais aussi du public et des experts - une capacité à refouler leurs émotions, faute de quoi ils peuvent perdre leur statut. Ainsi de cette jurée qui, suite à un malaise vagal en audience, a été remplacée par sa suppléante. À cet égard, la comparaison avec d'autres dispositifs de participation est édifiante. "Si chaque dispositif participatif propose aux "citoyens" appelés à y prendre part une forme de subjectivation ancrée sur des épreuves affectives auxquelles ils peuvent tout aussi bien se conformer que résister", le jury populaire constitue sans doute "un cas limite» (L. Blondiaux et C. Traïni, 2018, p. 37) tant la conformité à l'encadrement judiciaire des émotions y est absolument requise.

Cela est valable a fortiori pour les magistrats. À partir des notions de statut professionnel ${ }^{36}$, de statut situé 37 et de charisme de groupe ${ }^{38}$, S. Bergman Blix et Å. Wettergren (2018a, p. 24) s'intéressent à la manière dont les dynamiques de pouvoir et de statut qui s'exercent à l'audience, influencent les émotions ressenties et exprimées, et les stratégies de gestion des émotions. Dans la justice en effet, le statut et le pouvoir des magistrats impliquent de la déférence à leur égard, notamment vis-à-vis du juge qui incarne la loi (p. 40). Les interactions entre juges professionnels et profanes (qu'il s'agisse des assesseurs ou du public) montrent parfois l'échec des négociations de statut qui impliquent l'usage explicite de ressources de pouvoir à l'audience ou lors des délibérations. Le procureur, lui, se situe dans une dépendance limitée et donc dans une relation de pouvoir négocié sur la police ; il combine pouvoir (conféré par l'État de droit) et statut (où l'enjeu est d'être apprécié des policiers) pour gérer les éventuelles résistances de la police.

35. William M. REDDY, "Against Constructionism: The Historical Ethnography of Emotions", Current Anthropology, 38 (3), 1997, p. 327-351.

36. Andrew Аввотт, «Status and Status Strain in the Professions », American Journal of Sociology, 86, 1981, p. 819-835.

37. Theodore D. Kemper, Status, Power, and Ritual Interaction, Farnham: Ashgate Publishing Limited, 2011.

38. Norbert Elias et John L. SCOTTSON, The Established and the Outsiders, Londres : Sage, 1994. 
Les hiérarchies et asymétries de statut et de pouvoir structurent également le rapport pratique et ordinaire à l'humour en audience : lorsque l'on est avocat, greffier ou procureur, il convient de ne pas rire si le juge dit sérieusement des choses que l'on trouve drôles (S. Roach Anleu et K. Mack, 2018, p. 195). L'humour comprend donc une dimension normative dans la mesure où il existe des règles pratiques situées et intériorisées sur qui peut faire de l'esprit, comment et à propos de quoi. L'étude australienne montre que les plaisanteries ou l'expression au second degré interviennent principalement à l'initiative du juge (ibid., p. 151), qui domine l'audience. Ces résultats convergent avec ceux de l'étude suédoise. La propension, dans les parquets suédois, à faire circuler des plaisanteries sur des juges naît probablement en partie de l'expérience de cette forme de domination et "sert à inverser momentanément le déséquilibre de pouvoir entre les deux professions" (S. Bergman Blix et Å. Wettergren 2018b, p. 200). L'humour traduit l'entre-soi et la solidarité du groupe ${ }^{39}$ qu'il s'agisse des procureurs par rapport aux juges ou des professionnels du droit vis-à-vis du public, puisqu'entre permanents de l'audience prévaut la règle pratique selon laquelle on s'arrête de blaguer quand le public entre.

\section{Les émotions conditionnent la légitimité de l'exercice de la justice aux yeux du public}

Pour T. Tyler ${ }^{40}$, une justice procédurale repose sur la confiance dans la capacité des autorités à servir au mieux la société ; le traitement respectueux et équitable de toute personne ; ainsi que sur la capacité des citoyens à exprimer leur point de vue et à s'impliquer dans le processus de décision. Cultiver la confiance des citoyens dans l'institution judiciaire est primordiale dans un État de droit pour garantir la légitimité de l'exercice de la justice ainsi que l'adhésion au système judiciaire. Selon S. Bergman Blix et Å. Wettergren (2018b), c'est l'enjeu de la quête d'objectivité et d'impartialité des magistrats. Dans les tribunaux suédois qu'elles ont étudiés, une grande attention est apportée aux principes de la justice procédurale et à l'accueil du public. Mais cette politique de «bons traitements » peut entrer en contradiction avec les effets implicites de l'idéologie du nouveau management public concernant l'organisation du temps sur la gestion des émotions. Le développement des politiques néolibérales et techniques qui accroissent le contrôle organisationnel, et l'efficience, conduit en effet à des relations moins personnalisées et plus détachées, à l'égard des prévenus surtout (chapitre 3). Des "dissonances émotionnelles " 41 peuvent résulter de l'impossibilité de concilier des objectifs contradictoires (bien accueillir les justiciables $v$ s maîtriser la durée des procès).

L'objectif politique de satisfaire aux principes de la justice procédurale semble être endossé par certains magistrats australiens qui utilisent l'humour comme un moyen de détendre l'atmosphère en salle d'audience (S. Roach Anleu et K. Mack,

39. Pour l'humour à l'égard des non-permanents de l'audience, dont les parties et le public, cf. Sharyn ROACH ANLEU et Kathy MACK, Performing Judicial Authority in the Lower Courts, op. cit; Sharyn ROACH ANLEU et Kathy МАCK, «Le quotidien des magistrats et le travail émotionnel », article cité.

40. Tom TYLER, Why People Obey the Law, op. cit.

41. Arlie Russel HochSCHILD, The Managed Heart. Commercialization of Human Feeling, Berkeley: University of California Press, 1983 - traduit sous le titre Le prix des sentiments. Au cœur du travail émotionnel, Paris : La Découverte, 2017. 
2018, p. 145). Cela est convergent avec les observations réalisées dans les juridictions suédoises où «il arrive que l'humour soit utilisé par les juges pour atténuer temporairement leur pouvoir. On peut supposer que cette constatation est conforme à l'évolution de l'approche de la fonction publique en matière de jugement, selon laquelle l'efficacité et le traitement des profanes prennent de plus en plus d'importance dans les évaluations des tribunaux", affirment S. Bergman Blix et Å. Wettergren (2018b, p. 204).

À l'audience, « rire aux dépens des profanes n'est généralement pas acceptable » par la communauté des professionnels du droit (ibid.). Cette règle pratique semble être aussi en vigueur quand l'humour est utilisé comme une sanction négative à l'encontre de l'accusé, tel que dans l'exemple suivant. Un juge fait de l'humour auprès de l'avocat de la défense en présence de son client, en prenant appui sur les torts de l'accusé. Cette tentative d'humour échoue auprès du conseil qui réagit en remettant l'accusé au premier plan et en soulignant le caractère sérieux de la situation pour lui. L'avocat, parce qu'il est en présence de celui qu'il a mandat de représenter, contraint le juge à revenir au cadre interactionnel classique des échanges en contexte judiciaire (S. Roach Anleu et K. Mack, 2018, p. 164-165).

\section{Conclusion}

La richesse des ouvrages et articles chroniqués atteste du caractère heuristique de ce courant de recherche et des perspectives qu'il ouvre. Si les travaux concernant les rapports entre droit, justice et émotions sont plus nombreux dans les pays de common law, ils suggèrent à quel point mener des recherches similaires dans les pays de droit romano-germanique complèterait la compréhension des modes d'exercice de la justice. Diversifier les pays où ce type d'analyse est mené, mais aussi ne pas se focaliser en priorité sur le pénal permettraient d'approfondir l'analyse des similarités et différences dans les émotions observées, exprimées et ressenties, et la gestion des émotions, en s'intéressant aux effets de l'organisation des tribunaux et du judiciaire (statut et pouvoir des divers professionnels du droit, rapport des magistrats au politique, confiance des citoyens), et aux variations socioculturelles quant au mode d'expression des émotions : cette dimension est seulement mentionnée dans l'introduction du dossier paru dans les Oñati Socio-Legal Series; S. Bergman Blix et A. Wettergren se réfèrent ponctuellement aux différences entre la Suède et certains pays de common law. Si de nombreux travaux se focalisent sur les scènes publiques et leurs coulisses, d'autres, plus rares, s'intéressent aux émotions dès la formation des professionnels - lieu de socialisation au script judiciaire sans passion; le thème Emotion and legal education est en l'occurrence peu abordé dans les publications recensées ${ }^{42}$. T. Maroney (2019) souligne à quel point "la mosaïque d'étude des émotions s'est enrichie, même si de nombreuses pièces sont encore manquantes ». Non seulement les émotions sont nombreuses, mais la diversité des institutions judiciaires est grande. C'est pourquoi les recherches doivent rendre compte des

42. Emma JonES, Emotions in the Law School: Transforming Legal Education Through the Passions, Londres : Routledge, 2020. 
différences en termes de genre, race, territoires, mais aussi de niveaux de juridiction, types de contentieux et de procédures, de modes de formations des magistrats et de cultures judiciaires.

Les sources empiriques mériteraient aussi d'être diversifiées en s'appuyant par exemple sur les opinions des juges sur certaines affaires, leurs discours, journaux intimes, lettres, biographies, ou encore sur les manières dont les médias donnent à voir leur travail. La pluridisciplinarité peut encourager l'émergence de nouvelles méthodes, qu'il s'agisse des recherches sur les mindfulness strategies mises en œuvre pour réduire les émotions ressenties ; de collaborations entre droit et neuroscience, ou du codage de vidéos retraçant les émotions visibles sur les visages des juges (microexpressions research). Ce qui importe, c'est que les enquêtes empiriques consolident et élargissent les fondements théoriques des travaux sur les rapports entre droit, politique et émotions. Dans l'ouvrage La démocratie des émotions, L. Blondiaux et C. Traïni ont inclus à côté de différentes institutions politiques les juridictions, en l'occurrence la cour d'assises, dans l'éventail des situations empiriques examinées. Des convergences sont apparues avec les autres publications discutées qui montrent le potentiel d'interdisciplinarité de l'étude des émotions.

À travers les publications chroniquées, il apparaît que les expériences émotionnelles sont étudiées le plus souvent du côté des professionnels du droit, en particulier les magistrats. C. Gissinger-Bosse fait utilement varier le prisme, puisqu'elle met l'accent sur celles des citoyens-jurés. Toutefois, les effets à plus long terme de ces expériences sur la manière dont les acteurs, en particulier les justiciables, se représentent la justice demeurent un point aveugle qu'il conviendrait d'éclairer. Dans ce domaine en effervescence, plusieurs volumes proposant des états des lieux ${ }^{43}$, annoncés comme étant à paraître en 2020, devraient permettre que d'autres chercheur.e.s s'emparent de ces questions.

43. Terry Maroney, Jody Lynee MadeIRA, Kathryn Temple et Emily Kidd White (eds.), Research Handbook on Law and Emotion, Cheltenham : Edward Elgar Handbook Series, 2020 (à paraître) ; Steven TudoR, Richard Weisman, Michael Proeve et Kate Rossmanith (eds.), Remorse and Criminal Justice. Multi-Disciplinary Perspectives, Londres : Routledge, 2020 (à paraître). 


\section{Bibliographie}

Bergman Blix Stina, Mack Kathy, Maroney Terry et Roach Anleu Sharyn, «Introducing an Interdisciplinary Frontier to Judging, Emotion and Emotion Work», Oñati Socio-Legal Series [en ligne], 9 (5), 2019, p. 548-556.

<https://doi.org/10.35295/osls.iisl/0000-0000-0000-1095>

Bergman Blix Stina et Wettergren Åsa, Professional Emotions in Court: A Sociological Perspective, Londres : Routledge, 2018a.

— «Humour in the Swedish Court: Managing Emotions, Status and Power", in Jessica Milner DAVIS et Sharyn RoACH ANLEU (eds.), Judges, Judging and Humour, Cham : Palgrave Macmillan, 2018b, p. 197-227.

BLONDIAUX Loïc et TRAÏNI Christophe, «Les émotions, angle mort et dimension essentielle de la participation politique ", in ID. (dir.), La démocratie des émotions, Paris : Presses de Sciences Po, 2018, p. 7-43.

Bоотн Tracey, «Family Violence and Judicial Empathy: Managing Personal Cross Examination in Australian Family Law Proceedings ", Oñati Socio-Legal Series [en ligne], 9 (5), 2019, p. 702-725.

<https://doi.org/10.35295/osls.iisl/0000-0000-0000-1037>

GENDron Maria et BARREtT Lisa F., "A Role for Emotional Granularity in Judging", Oñati Socio-Legal Series [en ligne], 9 (5), p. 557-576.

<https://doi.org/10.35295/osls.iisl/0000-0000-0000-1087>

GISSINGER-Bosse Célia, «L'instruction des émotions. Le jury populaire dans l'institution judiciaire ", in Loïc BlONDIAUX et Christophe TRAÏNI (dir.), La démocratie des émotions, Paris : Presses de Sciences Po, 2018, p. 121-144.

LEBEN Steve, «Exploring the Overlap between Procedural-Justice Principles and Emotion Regulation in the Court Room », Oñati Socio-Legal Series [en ligne], 9 (5), 2019, p. 852-864.

$<$ https://doi.org/10.35295/osls.iisl/0000-0000-0000-1068>

Maroney Terry A., « Empirically Investigating Judicial Emotion », Oñati Socio-Legal Series [en ligne], 9 (5), 2019, p. 799-830.

<https://doi.org/10.35295/osls.iisl/0000-0000-0000-1089>

Milner Davis Jessica et RoACH Anleu Sharyn, « Thinking About Judges, Judging and Humour: The Intersection of Opposites ", in Jessica MiLNER DAVIS et Sharyn ROACH ANLEU (eds.), Judges, Judging and Humour, Cham: Palgrave Macmillan, 2018, p. 22-59.

Moran Leslie J., "The Wit of Judge Rinder: Judges, Humour and Popular Culture", Oñati Socio-Legal Series [en ligne], 9 (5), 2019, p. 771-798. <https://doi.org/10.35295/osls.iisl/0000-0000-0000-1036>

ROACH-ANLEU Sharyn et MACK Kathy, "A Sociological Perspective on Emotion Work and Judging », Oñati Socio-Legal Series [en ligne], 2019, 9 (5), p. 831-851.

<https://doi.org/10.35295/osls.iisl/0000-0000-0000-1032>

— «Judicial Humour and Inter-Professional Relations in the Courtroom ", in Jessica MiLNER DAVIS et Sharyn ROACH ANLEU (eds.), Judges, Judging and Humour, Cham : Palgrave Macmillan, 2018, p. 159-178. 
RossNer Meredith, «Storytelling Rituals in Jury Deliberations », Oñati Socio-Legal Series [en ligne], 9 (5), 2019, p. 747-770. <https://doi.org/10.35295/osls.iisl/0000-0000-0000-1038>

SCHREver Carly, Hulbert Carol A. et Sourdin Tania, « The Psychological Impact of Judicial Work: Australia's First Empirical Research Measuring Judicial Stress and Wellbeing ", Journal of Judicial Administration, 28 (3), 2019, p. 141-168.

SPOERER Mathilde, «L'institutionnalisation de la sensibilité indigène. La participation des autochtones au Chili », in Loïc BlOndiAUX et Christophe TraïNI (dir.), La démocratie des émotions, Paris : Presses de Sciences Po, 2018, p. 145-166.

ToY-Cronin Bridgette, «Leaving Emotion Out: Litigants in Person and Emotion in New Zealand Civil Courts », Oñati Socio-Legal Series [en ligne], 2019, 9 (5), p. 684-701. $<$ https://doi.org/10.35295/osls.iisl/0000-0000-0000-1034>

\section{- Les autrices}

Politiste, Laurence Dumoulin est chargée de recherche CNRS au laboratoire PACTE à l'Institut d'Études Politiques de Grenoble. Elle développe des recherches au carrefour de la sociologie du droit et de la justice et de l'analyse de l'action publique. Elle a notamment publié :

— «Le bracelet électronique : nouvelles économies pénales de la présence», in René LÉVY, Laurence DUMOULIN, Annie KENSEY et Christian LICOPPE (dir.), Le bracelet électronique : action publique, pénalité et connectivité, Genève : Éditions Médecine \& Hygiène, coll. « Déviance et société », 2019 ;

- Les audiences à distance. Genèse et institutionnalisation d'une innovation dans la justice (avec Christian LICOPPE), Paris : LGDJ Lextenso éditions, coll. « Droit et Société », 2017 ; — «Actor Network Theory and CCTV Development» (avec Anne-Cécile DoUILLET), in Dominique ROBERT et Martin DUFRESNE (eds.), Actor-Network Theory and Crime Studies: Explorations in Science and Technology, Farnham : Ashgate Publishing, 2015.

Cécile Vigour est chargée de recherches au CNRS en sociologie et science politique. Croisant enquêtes qualitatives et quantitatives, ses travaux étudient les transformations de la justice en lien avec les réformes globales de l'État, du double point de vue des professionnels et citoyens. Son habilitation portait sur : « La justice à l'épreuve de la gestion publique. Sociologie de la gestionnarisation des organisations publiques » (2019). Parmi ses publications :

— " "On a soif d'idéal!" Des critiques à la hauteur des attentes des citoyens. Un éclairage à partir d'entretiens collectifs" (avec Laurence DuMOULIN), in Soraya AMRANIMEKKI (dir.), Et si on parlait du justiciable du XXI siècle ?, Paris : Dalloz, 2019 ;

— «Les représentations sociales des peines en France. Une approche par entretiens collectifs " (avec Virginie GAUTRON), in Diane BERNARD et Kevin LADD (dir.), Les sens de la peine, Bruxelles : Presses de l'Université Saint-Louis, 2019 ;

- Réformes de la justice en Europe. Entre politique et gestion, Louvain-La-Neuve: De Boeck Supérieur, coll. « Ouvertures sociologiques », 2018. 